\title{
LAS NOCIONES DE POIESIS, PRAXIS Y TECHNÉ EN LA PRODUCCIÓN ARTÍSTICA
}

The ideas of Poiesis, Praxis and Techné in Artistic Production

\section{H. Marcelo Zambrano U.}

ISSN (imp): 1390-4825

ISSN (e): 2477-9199

Fecha de recepción: 04/02/19

Fecha de aceptación: 05/03/19 


\section{Resumen:}

El presente artículo inicia su desarrollo a partir del problema estético de la experiencia de la creación artística desde la creación artística desde la producción. Fundamenta su estructura desde las definiciones de poiesis, techné y praxis propuestas por Giorgio Agamben y articula su planteamiento de superación de la estética con la teoría decolonial y la liberación de la aísthesis, con el objetivo de establecer la noción de creación artística como una forma de producción poiética que posibilite la creación de mundos dentro de este mundo.

\section{Palabras clave:}

poiesis, praxis, techné, práctica artística

\section{Abstract:}

The present article develop the aesthetic problem of the artistic creations experience from the problematic of the This article develops the problem of aesthetic in artistic creations from the production point of view. It's structure is based on the definition of poiesis, techné and praxis as proposed by Giorgio Agamben, and enunciates his approach about overcoming aesthetics with decolonial theory and the liberation of aísthesis, with the aim of defining the notion of artistic creation as a form of poietic production that enables the creation of worlds within this world.

\section{Key Words:}

poiesis, praxis, techné, artistic practice

\section{Biografía del autor:}

H. Marzelo Zambrano, (Quito, Ecuador, 1972). Docente en varias universidades. Magíster en Estudios de la Cultura por la Universidad Andina Simón Bolívar, sede Quito, y Diplomado en Teorías del Arte Contemporáneo por la Pontificia Universidad Javeriana, de Bogotá, Colombia. Licenciado en Artes Plásticas por la Universidad Central del Ecuador; Técnico Superior en Diseño Gráfico por el Instituto Metropolitano de Diseño de Quito. Actualmente interesado en la investigación sobre temas relacionados con arte contemporáneo, tecnología, arte y ciencia, y prácticas curatoriales. 


\section{Introducción}

Giorgio Agamben (1970), al inicio del libro El hombre sin contenido, recuerda la crítica de Nietszche a Kant, cuando ubicaba el problema estético del arte en la mirada y perspectiva del espectador. Nietszche cuestionaba la definición kantiana de belleza desinteresada y el énfasis que el filósofo hacía en la contemplación del espectador; en su lugar proponía un giro hacia la experiencia del artista, es decir, el arte y el problema estético desde el punto de vista del creador, desde el "espectador desinteresado al artista interesado" (p.11).

Agamben atraviesa una profunda reflexión a partir de definiciones y articulaciones entre términos griegos, latinos y nociones occidentales sobre arte y estética, se enfoca en la experiencia creativa del artista y la manera en la que sobreviviría en una sociedad atravesada por la idea de una práctica artística basada casi exclusivamente en la experiencia y su relación con la producción poiética.

El presente texto parte de este punto, el giro del problema estético desde el espectador hacia el "hacedor", desde la experiencia que provoca la creación hacia la problemática de la producción. El texto toma las bases trazadas por Agamben con relación a las definiciones de poiesis, technéy praxis, profundiza en las articulaciones entre estos términos y enlaza la idea de Agamben de superación de la estética con los planteamientos poscoloniales de liberación de la aisthesis y creación artística como una forma de producción poiética que permita la creación de mundos dentro de este mundo.

\section{Poiesis y praxis}

En la antigua Grecia, el término poiesis definía al hacer productivo del ser humano. Tiene su raíz en la palabra poiein que significa hacer, fabricar; pero posee características que diferencian esta actividad de otras formas de producción y se distancia significativamente de las actuales definiciones de formas de hacer.

Para Platón (1988a) la poiesis se establece como "toda causa que haga pasar cualquier cosa del no-ser al ser" (p.252, 205b), es decir, toda actividad que permita la "producción" de algo desde su condición de no-existencia hacia la presencia. En la antigüedad clásica, este sentido amplio de la palabra poiesis abarcó diversas posibilidades del hacer productivo, como las actividades del artista, del artesano o la simple fabricación de algo, pero también todo lo que, de manera espontánea, la naturaleza trae a la presencia.

En este sentido, Aristóteles (1995) establece una correspondencia entre el principio de entrada a la presencia de las cosas y las formas en las que se producen las cosas. Así, lo que ha sido producido por la naturaleza tiene su principio en sí mismo, mientras lo que ha entrado a la presencia a partir de la actividad productiva del ser humano posee este principio fuera de sí mismo (p.133, 193a30). Esta diferencia determinó al segundo tipo de actividad productiva, es decir, al mantener externamente su principio de origen, necesitaba una causa de producción externa, a diferencia de la naturaleza que "producía" de forma espontánea, lo que permitió el establecimiento de una actividad productiva basada en procesos racionales externos y, por tanto, identificados con fines que se encontraban fuera de la propia actividad.

Agamben (1970) señala que todas estas actividades productivas pertenecían a la noción de poiesis, es decir, toda actividad realizada por el ser humano, como, los objetos fabricados por un artesano o la producción realizada por artistas -pinturas, esculturas o poesías(p.98), mantienen presente su definición como acto que permite el paso del no-ser a la presencia.

La definición griega de poiesis implicaba la existencia de otra noción conectada, la praxis. Tanto la poiesis como la praxis se relacionaban con la idea de hacer, es decir, con la producción. Sin embargo, existía un elemento fundamental a tomar en cuenta que permitía acercar y a la vez distanciar ambos términos. La poiesis, según se señaló, definía toda actividad que tenía como objetivo el paso desde la no-existencia hacia la presencia, y ubicaba en el centro de esta actividad a la misma producción de esta presencia. Mientras que la praxis, señala Agamben (1970), ubica en su centro la idea de voluntad determinada a través de la acción (p.112) y se encuentra basada en la empeiria, en la experiencia. Con el objetivo de explicar esta diferencia, es preciso desarrollar la idea de trabajo y su relación con las actividades de producción.

Así, en la antigüedad, la noción de trabajo se definía a partir de una compleja configuración conceptual. Para empezar, señala Agamben, el trabajo no pertenecía directamente a la vida material del ser humano, ya que todo el trabajo necesario para la vida de los individuos era realizado por sirvientes y esclavos. La sumisión a esta necesidad hubiera implicado equiparar la condición humana con la condición animal, que los obligaba a la 
búsqueda permanente del sustento vital para sobrevivir, algo que no se encontraba en correspondencia con la idea de hombre libre (p.113).

Las actividades se dividen, entonces, entre las necesarias para la vida -que poseían una naturaleza servil-y las que no eran necesarias y debían ser realizadas por hombres libres, usualmente caracterizadas por un mayor uso del intelecto y la reflexión. De esta manera, la configuración de la idea de trabajo con relación a la noción de producción se establece a partir del esfuerzo físico de la actividad. A mayor esfuerzo físico en la actividad, menor consideración por parte de los hombres libres y mayor cercanía con las actividades usualmente asignadas a los esclavos. Se establece así la diferencia entre trabajo manual y trabajo intelectual. Por esta razón, incluso el mismo Aristóteles entendía la diferencia entre poiesis y praxis como una distancia jerárquica que ubicaba a la poiesis sobre la praxis.

Posteriormente, según señala Agamben (1970), el pensamiento teológico cristiano identificó al Ser Supremo como una especie de actus purus y modificó, a partir de esta identificación, la metafísica occidental al extender esta interpretación a la consideración del ser como un efecto de la realidad a través de la acción, lo que provocó la convergencia de la poiesis y la praxis en una sola idea indistinguible (p.114). Así, la idea de trabajo identificada como una de las condiciones con menor valor jerárquico en términos de actividad productiva por los griegos, según se señaló, se establece como criterio central para la definición de la vida material del ser humano.

La condición vital de los individuos se establece casi exclusivamente como práctica, es decir, como actividad productora de la condición vital del ser humano a partir de la idea material de trabajo. El ser humano "entendido como ser vivo (animal) que trabaja (laborans) y, en el trabajo, se produce a sí mismo y se asegura el dominio de la tierra", escribe Agamben (p.115), en otras palabras, el ser humano adopta una condición productiva en el mundo, una condición práctica que definirá consecuentemente su condición humana a través de la praxis.

Este proceso, señala Agamben, eclipsa la esfera de la poiesis al vincular la idea de trabajo con la de producción material, desde la esfera de la técnica, lo que impide la posibilidad de diferenciar la producción poiética de la praxis.

\section{Poiesis y Techné}

La palabra techné refería un amplio abanico de actividades relacionadas con habilidades y destrezas necesarias para la producción de algo dentro de una dimensión técnica. Usualmente, el término se traduce como arte, técnica u oficio y se define por estar basado en la aplicación de conocimientos técnicos y habilidades enlazadas a objetivos conscientes y principios racionales (Flyvbjerg, 2001, p.56).

Debido a su tendencia de origen hacia la producción, la techné pertenece a la esfera de la poiesis, por tanto, la noción de techné debería entenderse a partir de sus características en tanto producción poiética. En este sentido, la techné podría ser pensada como una producción que permite el desvelamiento de la verdad en el paso del no-ser hacia la presencia, a partir de las posibilidades que permite el dominio del conocimiento racional de cómo se producen las cosas.

De esta forma, si la noción de techné integraba las amplias posibilidades de la esfera de la poiesis, entonces, abarcaba también diversas actividades prácticas y oficios, desde hacer zapatos hasta construir barcos, incluyendo la pintura, la escultura y, a partir de las posibilidades de control del conocimiento productivo, lo que actualmente se consideran ciencias, como las matemáticas y la astronomía.

Esta diversa comprensión de la capacidad del ser humano para producir se torna compleja en el siglo XVIII. En principio, los diferentes intentos por clasificar la producción dentro del ámbito de la poiesis incluían y excluían diferentes prácticas y actividades; así, tanto las actividades manuales que actualmente se consideran como oficios artesanales, como las disciplinas que se contemplan como ciencias, en cierto momento formaron parte de la clasificación de las artes (Tatarkiewickz, 2001, p.85). Sin embargo, esta noción amplia de elementos y actividades relacionadas tanto con la poiesis como con la techné se desarticula. Así, se forman tres espacios distintos y perfectamente delimitados, las ciencias, las artesanías y las bellas artes, desde los cuales la idea de producción empezará a reconfigurarse (Zambrano, 2016, p.113).

Agamben (1970) identifica que esta separación se establece de forma paralela al desarrollo técnico, científico y filosófico del siglo XVIII, lo que provocó una transformación en la forma de las cosas que la producción 
poiética trae a la presencia y define a la poiesis a partir de una doble consideración: lo realizado por la técnica en forma de productos -serialidad industrial a partir de la posibilidad de reproductibilidad- y lo realizado por la estética en forma de obras de arte-originalidad en forma de acción creativa- (p.100).

En este punto aparecen las actuales ideas de arte y de producción artística dentro de la esfera estética. $\mathrm{El}$ arte se convierte en un espacio disciplinar autónomo y cerrado, articulado con los emergentes conceptos ilustrados de estética y belleza racional que, junto a la visión de una práctica artística diferente a la propuesta por la producción poiética, se dispone como una actividad netamente práctica.

Agamben identifica esta transformación con la entrada de la obra de arte (poiesis) en la dimensión estética, esfera privilegiada que sostiene como fundamento la idea de que el trabajo manual y el trabajo intelectual no se encuentran separados y que el acto poiético, por tanto, mantiene aún su unicidad (p.101). Mientras que la producción técnica, continúa Agamben, por el contrario, se mantendría sobre el terreno de la extrema división del trabajo.

Así, la noción de techné como parte de la poiesis desaparece, deja de lado la existencia de unicidad productiva en la poiesis - unidad que no discrimina la producción estética de la técnica- la divide y permite, por tanto, el aparecimiento de nociones diferentes de producción técnica y de producción poiética, que, según se señaló, corresponden paralelamente tanto al eclipse de la noción de techné, como a la convergencia entre la poiesis y la praxis señaladas por Agamben (1970, p.114).

Propuesta de creación de nuevos mundos a partir de la noción de poiesis

La poiesis es un tipo de producción que permite el paso del no-ser a la presencia, admite el develamiento de un espacio de verdad (Agamben, 1970, p.114) y se la puede entender como un devenir que dilata lo que se encuentra en la posibilidad de lo que puede llegar a ser (Jagodzinski y Wallin, 2013, p.85).

En este sentido, lo que puede llegar a ser de otra manera, escribe Aristóteles (2005), puede producirse a partir de la poiesis (fabricación) o desde la praxis (realización), que no se incluyen una en la otra, ya que la fabricación no es realización ni la realización es fabricación, señala Aristóteles, y debido a que la techné (producción a partir de un conocimiento productivo) se encuentra dentro de las posibilidades de la construcción, y la construcción es fabricación, la techné se entendería como una disposición acompańada de razón verdadera, relativa a la fabricación (p.185, 1140a).

Heidegger (1997), en su reflexión sobre la esencia de la técnica, ubica a la techné como el hacer de las artes y la artesanía, como un saber elevado perteneciente a la producción poiética, incluso cercana a la episteme (conocimiento teórico) hasta el punto de confundirse -ambos términos- en el acto de conocer. El conocer abre, continúa Heidegger, desoculta (p.121), y este desocultamiento, que se presenta como una "una conexión de efectos de fuerzas calculables" y "constataciones exactas", como desocultamiento de la verdad, tiene como resultado la persistencia del "peligro de que en todo lo exacto se retraiga lo verdadero" (p.137).

El peligro potencial advertido tomó forma en las interpretaciones y traducciones de la misma palabra techné. Así, además de tomar el sentido de oficio, habilidad, arte o experticia, en ciertos contextos, el término techné se traduce como artificio, ardid, truco o trampa (Angier, 2010, p.6).

Se entiende, entonces, que esta relación con la verdad propia de la poiesis y, por ende, de la techné, hubiera causado algo de incomodidad en la antigüedad. Platón (1988b) advirtió esta posibilidad, temiendo la destrucción de su ciudad debido a esta particular disposición productiva de la poiesis, exhortando finalmente por la expulsión y el exilio de la poesía (p.476, 607b).

Ahora bien, según lo señalado, la convergencia de la poiesis con la praxis permitió la división de las posibilidades de producción poiética, y provocó la conformación de la dimensión estética y el ingreso de la obra de arte en esta dimensión. Así, la estética se conforma eclipsando la noción amplia de techné, dirigiendo su configuración hacia la clausura de la posibilidad de acercamiento a la producción de un espacio de verdad. De esta manera, se desarrolla una esfera estética autónoma e independiente que expulsa de su configuración interna cualquier elemento propio de la vida del ser humano, autonomía que sería posteriormente confrontada por la agenda política de las vanguardias históricas (Bürger, 1974, p.63). 
En este escenario, el peligro que advertía Platón se presenta tanto en la búsqueda por unir el arte y la vida de los artistas modernos, como en la imploración de Pigmalión a la diosa, para lograr la felicidad a partir de la creación poiética, en ambos casos, la búsqueda de un camino que conduzca hacia nuevos espacios de verdad en la producción que permite la poiesis, es clara. Las posibilidades, desde estos espacios de verdad que la producción poiética admite, permiten pensar en la construcción -en tanto fabricación, en tanto technéde nuevos entornos para ser ocupados a partir de la producción artística.

Agamben (1970) observa la ligereza con la que esta condición productiva original se relaciona con la obra de arte en tanto fundamento de un espacio de verdad, y, desde esta perspectiva, propone que sea el paso del noser hacia la presencia el que permita el develamiento de un espacio de verdad, y edifique un mundo en el cual el ser humano pueda habitar sobre la tierra, a partir de la superación de la dimensión estética (p.114).

Sin embargo, a pesar de que esta intención no se establezca radicalmente como un intento por superar la estética, actualmente existen posiciones tanto epistemológicas como prácticas que se encuentran dispuestas a reconfigurar las condiciones de la obra de arte, es decir, la producción poiética. Estas posiciones descansan sobre las bases de las teorías decoloniales, que proponen la identificación de ciertos límites y características de las prácticas artísticas en la modernidad y las asocian con los patrones de poder que las configuraron.

Aníbal Quijano (2007) identifica un patrón de poder fundamentado en el sistema económico capitalista que habría configurado la mayoría de los elementos constitutivos de la vida del ser humano (p.94). Este patrón de poder, al que Santiago Castro-Gómez (2004) ubica desde el siglo XVIII, determina principalmente la manera en la que se conforman los sentidos de las cosas del mundo. Así, uno de los ámbitos al que este patrón de poder colonial afecta significativamente es el de la estética.

En este sentido, Walter Mignolo (2012) propone la revisión del antiguo término griego de aisthesis, que se relacionaba directamente con el ámbito de la percepción sensible y fuera utilizado en el siglo XVIII como raíz de la actual palabra estética. Esta revisión planteada por Mignolo, concibe la noción de aisthesis como un espacio colonizado por la estética moderna que determina tanto su configuración (epistemológica, ontológica) como lo que se considera y no se considera arte (p.9). Propone, por tanto, la emergencia de una estética decolonial, que posibilite la liberación de la idea de estética (más cercana a la de aisthesis) de este patrón colonial con el fin de desobedecer las reglas, normas y valores impuestos por la estética moderna.

Esta desobediencia estética, que desde ciertas perspectivas se presentaría como estético-epistémica, por su relación y cercanía con la colonialidad del saber (Bouhaben, 2018, p.190), plantea la liberación de las subjetividades $\mathrm{y}$, a partir de esta remisión, la producción (cercana a la idea de poiesis) de sentidos, emociones, reflexiones y elementos simbólicos que permitan la emergencia de nuevas formas de ver y entender el mundo fuera de este patrón de poder colonial.

En estesentido, las reflexionesylos planteamientos decoloniales sobre la práctica artística abordados en la actualidad por teóricos, artistas, diseñadores y artesanos se encuentran definidos por el regreso de la atención hacia lo que se señaló como producción poiética, es decir, un espacio en el que la presencia toma lugar desde la noexistencia, no como el desvelamiento de una forma de verdad, sino como el devenir del ser hacia la presencia desde la emergencia de una multiplicidad de verdades.

La producción artística, desde esta perspectiva, supera las oposiciones jerárquicas binarias entre lo bello y lo feo, lo trágico y lo cómico, lo elevado y lo bajo, para entender, desde un principio de dualidad no-excluyente, la existencia de otras opciones que se enlacen con modelos estético y epistémicos diferentes (Tlostanova, 2012, p.66). Esta producción, que separaría la convergencia entre poiesis y praxis y provocaría una suerte de liberación de la aisthesis, no tiene relación únicamente con la productividad racional (techné) o con la voluntad de acción (praxis) sino con ese todo mayor que permite el devenir de la presencia en la producción, fuera de los cánones estéticos modernos.

La producción de la artesanía y el diseño, entendidos como oficios separados de la idea de producción poiética en el siglo XVIII, también se reconfiguran en la actualidad. El diseño ontológico, por ejemplo, entiende la importancia de la producción poiética de objetos, estructuras, políticas o discursos, con el objetivo de crear - develar- otras formas de ser. Es decir, a partir de la atención que se preste a la producción desde la poiesis, es posible pensar que el mundo está siendo diseñado por el 
mismo ser humano y que el mundo, a su vez, diseña al ser humano (Escobar, 2016, p.27). En otras palabras, el ser humano deviene como tal en función de su producción poiética, externamente al patrón de producción técnica o estética impuesta por el sistema de poder colonial.

Resulta entonces necesario repensar la techné y la poiesis al articularlas al pensamiento decolonial como la posibilidad de unir el arte y la vida, como una estrategia para sobrevivir creando mundos dentro de un único mundo impuesto, desde el continuo develamiento de la presencia del ser; pero ya no desde lo pensado por la estética o por la epistemología modernas, sino desde las posibilidades poiéticas que permiten la emergencia de algo desde el no-ser, desde la no-existencia, desde lo que aún no ha sido pensado.

Agamben (1970) esperaba "salir del pantano de la estética y de la técnica para devolverle su dimensión original a la condición poética del ser humano" (p.110). Queda restituirle al arte su posibilidad de abarcar y producir nuevamente el mundo.

\section{Referencias}

Agamben, G. (1970). El hombre sin contenido. Barcelona: Ediciones Áltera.

Angier, T. (2010). Techné in Aristotle's Ethics. Crafting the Moral Life. Londres: Continuum.

Aristóteles (2005). Ética a Nicómaco. Madrid: Alianza Editorial.

Aristóteles. (1995). Física. Madrid: Gredos.

Bouhaben, M. (2018). "La investigación artística eurocéntrica y su descolonización estético-epistémica”. En Rodriguez, A. y Alsina, P. "Arte e investigación”. ArtNodes. 21. 187-196.

Bürger, P. (1974). Teoría de la vanguardia. Barcelona: Ediciones Península.

Castro-Gómez, S. (2004). La Hybris del punto cero. Ciencia, raza e ilustración en la Nueva Granada (17501816). Bogotá: Universidad Javeriana.

Escobar, A. (2016). Autonomía y Diseño. Sobre la construcción de lo comunal. Popayán: Editorial Universidad del Cauca.
Flyvbjerg, B. (2001). Making Social Science Matter. Why social inquiry fails and how it can succeed again. Cambridge: Cambridge University Press.

Heidegger, M. (1997). Filosofía, ciencia y técnica. Santiago de Chile: Editorial Universitaria.

Jagodzinski, J. y Wallin, J. (2013). Art-Based-Research. A Critique and a Proposal. Rotterdam: Sense Publishers.

Mignolo, W. y Gómez, P. (2012). Estéticas y opción decolonial. Bogotá: Universidad Distrital Francisco José de Caldas.

Platón. (1988a). Diálogos vol.III. Fedón. Banquete. Fedro. Madrid: Gredos.

Platón. (1988b). Diálogos vol.IV. República. Madrid: Gredos.

Quijano, A. (2007). "Colonialidad del poder y clasificación social”. En Castro-Gómez, S. y Grosfoguel, R. (2007). El giro decolonial. Reflexiones para una diversidad epistémica más allá del capitalismo global. Bogotá: Siglo del Hombre Editores. pp. 93-125.

Tatarkiewicz, W. (2001). Historia de seis ideas. Arte, belleza, forma, creatividad, mimesis, experiencia estética. Madrid: Teknos.

Tlostanova, M. (2012). "La Aesthesis trans-moderna en la zona fronteriza eurasiática y el anti-sublime decolonial”. En Mignolo, W. y Gómez, P. (2012). Estéticas y opción decolonial. Bogotá: Universidad Distrital Francisco José de Caldas. pp. 53-89.

Zambrano, M. (2016). La investigación en el arte -la relación arte y ciencia, una introducción. En INDEX \#01. Quito: PUCE. 110-116 